\title{
Virus Infection
}

3 Candelaria Vergara ${ }^{1}$, Priya Duggal ${ }^{1}$, Chloe L. Thio ${ }^{2}$, Ana Valencia ${ }^{2,3}$, Thomas R. O’Brien ${ }^{4}$,

4 Rachel Latanich ${ }^{2}$, Winston Timp ${ }^{2}$, Eric O. Johnson ${ }^{5}$, Alex H. Kral ${ }^{5}$, Alessandra Mangia ${ }^{6}$, James

5 J. Goedert $^{7}$, Valeria Piazzola ${ }^{6}$, Shruti H. Mehta ${ }^{1}$, Gregory D. Kirk ${ }^{1}$, Marion G. Peters ${ }^{8}$, Sharyne

6 M. Donfield ${ }^{9}$, Brian R. Edlin ${ }^{10}$, Michael P. Busch ${ }^{11}$, Graeme Alexander ${ }^{12}$, Edward L. Murphy ${ }^{11}$,

7 Arthur Y. Kim ${ }^{13}$, Georg M. Lauer ${ }^{14}$, Raymond T. Chung ${ }^{14}$, Matthew E. Cramp ${ }^{15}$, Andrea L.

$8 \operatorname{Cox}^{2}$, Salim I. Khakoo ${ }^{16}$, Hugo R. Rosen ${ }^{17}$, Laurent Alric ${ }^{18}$, Sarah J. Wheelan ${ }^{1,2}$, Genevieve L.

9 Wojcik $^{19}$, David L. Thomas ${ }^{2}$ and Margaret A. Taub ${ }^{1}$.

$10{ }^{1}$ Johns Hopkins University, Bloomberg School of Public Health, Baltimore, MD. USA.

112 Johns Hopkins University, School of Medicine, Baltimore, MD, USA

$12{ }^{3}$ Universidad Pontificia Bolivariana, Medellin, Colombia.

$13{ }^{4}$ Division of Cancer Epidemiology and Genetics, National Cancer Institute, National Institutes 14 of Health, Bethesda, MD, USA

$15 \quad{ }^{5}$ RTI International, Research Triangle Park, NC. USA

$16{ }^{6}$ Liver Unit IRCCS “Casa Sollievo della Sofferenza”, San Giovanni Rotondo, Italy

$17{ }^{7}$ Division of Cancer Epidemiology and Genetics, National Cancer Institute, National Institutes 18 of Health, Bethesda, MD. USA

$19{ }^{8}$ Department of Gastroenterology, University of California, San Francisco, CA., USA.

$20 \quad{ }^{9}$ Rho, Inc., Chapel Hill, NC, USA

$21{ }^{10}$ Centers for Disease Control and Prevention, Atlanta, GA., USA

$22{ }^{11}$ University of California San Francisco and Vitalant Research Institute, San Francisco, CA., 23 USA 
$24{ }^{12}$ University College London Institute for Liver and Digestive Health, The Royal Free Hospital, 25 London, UK.

$26{ }^{13}$ Division of Infectious Diseases, Department of Medicine, Massachusetts General Hospital and 27 Harvard Medical School, Boston, MA. USA

$28{ }^{14}$ Liver Center and Gastrointestinal Division, Department of Medicine, Massachusetts General 29 Hospital and Harvard Medical School, Boston, MA.,USA

$30 \quad{ }^{15}$ South West Liver Unit, Plymouth, PL6 8DH, UK.

$31{ }^{16}$ University of Southampton, Southampton General Hospital, Southampton, UK.

$32{ }^{17}$ University of Colorado, Aurora, CO., USA

$33{ }^{18}$ Department of Internal Medicine and Digestive Diseases, Centre Hospitalier Universitaire 34 Rangueil, UMR 152, Institut de Recherche pour le Développement Toulouse 3 University, 35 France.

$36{ }^{19}$ Department of Genetics, Stanford University School of Medicine, Stanford, CA., USA

37 Short title

38 Fine-mapping of IFNL Locus for HCV clearance

39 Corresponding Author

40 Candelaria Vergara, $\mathrm{MD}, \mathrm{PhD}$

41725 N. Wolfe Street, Suite 211

42 Baltimore, Maryland 21205

$43 \quad$ Email: cvergar2@jhmi.edu

44 Disclosures: All authors declare that they have no conflict of interest and nothing to disclose. 45 
Funding: R01 013324, R01-HD-41224, RO1-AI148049-21， R21AI139012, U19-AI082630,

47 U01-AI131314, R01-DA033541, U19-AI066345, DA12568, DA036297, R01-DA09532, R01DA11860, N02CP91027, H79TI12103, R01DA16159, R01DA21550, R01-HD-41224.

49

50

51

52

53

54

55

56

57

60

61

62

63

64

65

66

67

68

\section{Abstract:}

Clearance of acute infection with hepatitis $\mathrm{C}$ virus (HCV) is associated with the chr19q13.13 region containing the rs368234815 (TT/ $\Delta \mathrm{G})$ polymorphism. We fine-mapped this region to detect possible causal variants that may contribute to $\mathrm{HCV}$-clearance. First, we performed sequencing of IFNL1-IFNL4 region in 64 individuals sampled according to rs368234815 genotype: TT/clearance $(\mathrm{N}=16)$ and $\Delta \mathrm{G} /$ persistent $(\mathrm{N}=15)$ (genotype-outcome concordant) or TT/persistent $(\mathrm{N}=19)$ and $\Delta \mathrm{G} /$ clearance $(\mathrm{N}=14)$ (discordant). 25 SNPs had a difference in counts of alternative allele $>5$ between clearance and persistence individuals. Then, we evaluated those markers in an association analysis of $\mathrm{HCV}$ clearance conditioning on rs368234815 in two groups of European (692 clearance/1 025 persistence) and African ancestry (320 clearance/1 515 persistence) individuals. 10/25 variants were associated $(\mathrm{P}<$ $0.05)$ in the conditioned analysis leaded by rs4803221 $\left(\mathrm{P}=4.9 \times 10^{-04}\right)$ and $\mathrm{rs} 8099917\left(\mathrm{P}=5.5 \times 10^{-}\right.$ $\left.{ }^{04}\right)$. In the European ancestry group, individuals with the haplotype rs368234815 $\Delta \mathrm{G} / \mathrm{rs} 4803221 \mathrm{C}$ were $1.7 \mathrm{x}$ more likely to clear than those with the $\mathrm{rs} 368234815 \Delta \mathrm{G} / \mathrm{rs} 4803221 \mathrm{G}$ haplotype $\left(\mathrm{P}=3.6 \times 10^{-5}\right)$. For another nearby SNP, the haplotype of rs368234815 $\mathrm{G} / \mathrm{rs} 8099917 \mathrm{~T}$ was associated with HCV-clearance compared to rs368234815 $\mathrm{G}$ /rs8099917G (OR: 1.6, $\mathrm{P}=1.8 \mathrm{x} 10^{-}$ ${ }^{4}$ ). We identified four possible causal variants: rs368234815, rs12982533, rs10612351 and rs4803221. Our results suggest a main signal of association represented by rs368234815, with contributions from rs4803221, and/or nearby SNPs including rs8099917.

\section{Introduction}


69 The outcome of the acute hepatitis $\mathrm{C}$ virus (HCV) infection is determined in part by host genetic 70 factors. Previous genome-wide association studies (GWAS) and meta-analyses have identified

71 significant associations of spontaneous clearance of $\mathrm{HCV}$ infection with several single 72 nucleotide polymorphisms (SNPs) in the region harboring 4 interferon- $\lambda$ genes (IFNL1, IFNL2, 73 IFNL3 and IFNL4) on chromosome 19q13.13 (1-3). Of particular importance is a dinucleotide 74 variant in the first exon of IFNL4, rs368234815 $(\Delta \mathrm{G} / \mathrm{TT})$, which causes a shift in the open 75 reading frame of the gene; the presence of the $\Delta \mathrm{G}$ allele at the variant position allows the 76 expression of a fully functional IFN $\lambda 4$ protein of 179 amino-acids $(4,5)$. This allele is 77 implicated in reduced HCV clearance (4). On the contrary, the TT allele is predicted to induce 78 nonsense-mediated mRNA decay and is associated with increased HCV clearance (4) (Figure 79 1, Top panel).

80 Despite the strong and replicated association, some HCV infected individuals carrying the 81 favorable genotype (TT) of rs368234815 do not clear the infection, while some patients with the

82 unfavorable genotypes spontaneously clear the infection $(4,6-10)$. This discordant IFNL 83 genotype with $\mathrm{HCV}$ infection outcomes are not explained by other determinants of 84 spontaneous clearance such as polymorphisms in other known HCV related genes, sex, or 85 HIV co-infection. Thus, we reasoned that other variants in the IFNL region may contribute to 86 the observed spontaneous clearance.

87

88 The IFNL1-IFNL4 region is under strong linkage disequilibrium (LD) which complicates the 89 identification of causal alleles (11). Moreover, sequencing of the region is limited by the 90 presence of genes with high homology that precludes the accurate assignment of reads to a 91 specific location $(12,13)$. In this study we sought to overcome these challenges and to identify 
92 variants that may contribute to clearance of $\mathrm{HCV}$ infection. We implemented two approaches to 93 identify variants with an association independent of rs368234815 (Figure 2). First, we 94 performed short-read sequencing analysis in a selected panel of individuals where the 95 rs368234815 genotype was either concordant or discordant with the expected HCV outcome 96 (clearance or persistence) using a sequencing strategy that allowed the precise assignment of the 97 reads to specific coordinates in the locus and accurate calling of the variants. Second, we performed conditional analysis in a large independent set of individuals of European and African ancestry evaluated for display of $\mathrm{HCV}$ spontaneous clearance for whom we had genotypes imputed to the 1000 Genomes Project (14). To identify potential causal variants in the

101 region we used well established statistical methods combining functional data from external 102 sources with the association and LD patterns from our datasets (Figure 2). We also interrogated 103 our dataset for association of two variants (rs1176648444 and rs4803217) that have been 104 identified as functionally relevant in the region.

105 Materials and Methods

106 Genetic structure of the IFNL region: The interferon lambda region spans $50 \mathrm{~Kb}$ of human 107 chromosome $19 q(15,16)$, Figure 3 . The 4 interferon- $\lambda$ genes seem to originate from gene 108 duplication events $(4,17)$ with IFNL2 and IFNL3 more closely related to each other than IFNL1 109 (4). IFNL1 and IFNL2 are transcribed from the positive strand with a coding region of 2,348 and 1101,445 basepairs (bp), with 5 and 6 exons, respectively. IFNL3 and IFNL4 have 6 and 5 exons 111 each, are transcribed from the negative strand and have a coding region of 1336 and 2543 bp, 112 respectively (Figure 3) (16).

\section{Short-read sequencing in panel of discordant and concordant individuals}


114 Individuals for IFNL Sequencing: Individuals included in this approach are part of the HCV

115 Extended Genetics Consortium $(2,18)$. Each individual study obtained consent for genetic testing

116 from their governing Institutional Review Board and the overall research was approved by the

117 Johns Hopkins School of Medicine Institutional Review Board (3). For this analysis we selected

11864 individuals based on the genotype of rs368234815 and HCV spontaneous

119 clearance/persistence status in a similar approach presented by Rauch et al for rs8099917 (1).

120 This included 45 individuals of African Ancestry (21 clearance/24 persistence) and 19

121 individuals of European Ancestry (9 clearance/10 persistence) (Table 1, Figure 1, Bottom panel).

122 These individuals were either concordant between genotype and HCV outcome (i.e. favorable

123 genotype of rs368234815 [TT/TT] and HCV spontaneous clearance or unfavorable genotypes

124 rs368234815 $[\Delta \mathrm{G} / \Delta \mathrm{G}]$ and HCV persistence) or were discordant (i.e. favorable genotype and

$125 \mathrm{HCV}$ persistence or unfavorable genotype and HCV clearance) (Table 1, Figure 1, Bottom 126 panel).

127 IFNL Sequencing: Because the region containing the four IFNL genes has low sequence 128 complexity, the alignment of short reads generated with standard high-throughput sequencing 129 methods is challenging (12); thus, we designed a targeted sequencing approach where the entire $13070.8 \mathrm{~Kb}$ region (chromosome 19:39721399-39792284, coordinates based on The Genome 131 Reference Consortium Human build 37-GRCh37-) was amplified in eight segments with 132 customized primers (Figure 3, Supplementary Table 1). This allowed alignment of reads 133 specifically to the region of origin, resulting in more confident detection of individual variants 134 across the whole region. Methods of DNA extraction and strategies for sequencing and 135 alignment are described in detail in Supplementary Material. 
136 Statistical Analysis of the sequenced panel: Counts of alternative (non-reference, non-ancestral)

137 alleles at each position of the sequenced region were generated to compare differences in single-

138 nucleotide variants (SNVs) between concordant and discordant individuals. We report all

139 positions where the difference in alternative allele count is at least 5. Given sample size

140 limitations, we did not perform formal statistical tests for differences between groups, but report

141 all positions for validation in imputation data. Customized scripts in R (https://www.r-

142 project.org/) were used to do the SNV analysis. Results of comparison of all variants included in

143 the analysis of the region is available upon request.

144 Conditional Analysis of an independent imputed dataset.

145 Individuals of the independent imputed dataset: We analyzed the rest of the individuals of the 146 HCV Extended Genetics Consortium $(2,18)$, corresponding to an independent set of 1835 147 individuals of African Ancestry (320 clearance/1515 persistence) and 1717 individuals of 148 European ancestry (692 clearance/1025 persistence). The $\operatorname{rs} 368234815 \Delta \mathrm{G}$ allele had a 149 frequency of 0.63 in the African ancestry group and 0.31 in the European ancestry group 150 (Table 1).

151 Genotyping and Imputation: Genotypes in this region were derived from a genome-wide 152 association study previously described $(2,3)$ and in Supplementary Material. For this analysis, 153421 high quality imputed variants in the IFNL region were used in African ancestry individuals 154 and 282 in European ancestry individuals.

155 Statistical Analysis of the independent imputed dataset: In each of the ancestry groups, we 156 performed an association analysis of dosage of the variants in the region conditioned on the 157 rs368234815 variant using an additive logistic regression model, adjusting for 3 principal 
158 components and HIV status using Mach2dat (19). Conditional analysis in the two ancestry

159 groups were meta-analyzed using the fixed effects inverse variance method in METAL software

160 (20). Given that this region has been highly replicated and to preserve power for detect

161 secondary signals in the fine mapping, a value of $\mathrm{P}<0.05$ was considered as significant (21).

162 Results from the imputation analysis were pulled to check for significance at any of the loci that

163 were identified based on alternative allele counts from the sequencing analysis. Candidate sites

164 with difference in the allele count and significance in the imputed dataset were carried forward

165 for the haplotype analysis.

166 Haplotype analysis: To further characterize the locus across populations, we conducted 167 haplotype association analyses. We calculated LD and constructed haplotypes based on the 168 candidate sites in the European ancestry and African ancestry populations. LD patterns in each 169 population were calculated using the algorithm from Gabriel et al (22) in Haploview (23) in the 170 imputed dataset. We performed haplotype analyses using the "haplo.stat" R package (24). We 171 assumed an additive model in which the regression coefficient represented the expected change 172 in the log odds of HCV clearance with each additional copy of the specific haplotype compared 173 with the reference haplotype.

\section{Identification of potential causal variants}

175 To identify variants that may be causal or have a regulatory function we refined the region 176 observed in a previous GWAS of HCV clearance (3). We used association summary statistics 177 from GWAS for those markers in the IFNL region, leveraged functional data and LD 178 information of the included markers and described a $99 \%$ credible set of variants using 179 PAINTOR $(25,26)$ as described in detail in Supplementary Materials. Aiming to optimize power 180 for this analysis, we included the complete dataset of the HCV Extended Genetic consortium 
181 comprising 3608 people from two ancestry groups: 1869 individuals of African ancestry (340

182 clearance/1529 persistence) and 1736 of European ancestry (701 clearance/1,035 persistence).

183 We considered PAINTOR predicted variants to be functional based on a posterior probability >

1840.1 , a threshold suggested previously (26). To investigate functional elements, the presence or 185 absence of overlap was determined by the UCSC Table Browser intersecting the calculated 186 credible set with the signal tracks described in Supplementary Materials.

\section{Analysis of functionally relevant variants}

188 Two markers (Rs4803217 and Rs1176648444) has been described as modulators of the 189 association given by rs368234815 (Supplementary Material). Given their potential functional 190 role, we evaluated their allele count in the sequenced dataset and the association of each variant 191 in the imputed dataset after conditioning on rs368234815. We also evaluated the residual 192 association after conditioning on both rs368234815 and rs1176648444 and the association of the 193 rs368234815- rs4803217 and rs368234815- rs1176648444 haplotypes in each population and 194 using the methods described in haplotype analysis, we constructed and evaluated association of 195 haplotypes based on the candidate sites common to European and African ancestry populations 196 incorporating these functionally relevant variants.

\section{Results}

198 When analyzing all individuals of the sequencing group (concordants and discordants), we 199 identified 25 positions (candidate SNVs) where the difference in the frequency of the alternative 200 allele was $>5$ between the individuals with clearance and persistence (Table 2). The identified 201 variants are located downstream of IFNL3-IFNL4 and in the intergenic regions between IFNL4202 IFNL2 and IFNL2-IFNL1 (Supplementary Figure 1). 
204 region in this dataset, we extracted the results of the 25 variants identified in the targeted 205 sequencing panel. From those, 2 variants were not present in both ancestry groups and 10 206 candidate variants were significantly associated $(\mathrm{P}$ value $<0.05)$ in the meta-analysis 207 (Supplementary Figure 1, Table 2). No other variants in the region was significantly associated 208 in the conditional analysis.

209 The 10 candidate variants are located in an 11.3Kb region (chr19: 39732501-39743821) 210 spanning $1.7 \mathrm{~Kb}$ downstream of IFNL3 and 4.3 Kb upstream of IFNL4 (Figure 4, Panel A-B and 211 Supplementary Figure 1). The association observed in this meta-analysis was driven mainly by 212 the contribution of the European ancestry samples (Table 2). In this group, nine of ten associated 213 variants have similar minor allele frequencies $(\sim 0.19)$ with the exception being rs4803222 214 (0.30). Rs4803221, a synonymous SNP in IFNL4 (NM_001276254: S (TCG) --> S (TCC)), had 215 the strongest association in the meta-analysis $\left(\mathrm{P}\right.$-value $\left.=4.86 \times 10^{-04}\right)$ and in the European 216 ancestry group $\left(\mathrm{P}\right.$-value $\left.=7.49 \times 10^{-06}\right)$. In the African ancestry population, the direction of the 217 effect is the same but no variants were significantly associated. The allele frequencies for seven 218 of ten variants were similar across the ancestry groups. However, at rs8107030, rs8099917 and 219 rs7248668 minor allele frequencies were lower in the African ancestry population $(0.04-0.06)$ 220 than in persons of European ancestry (0.19) (Table 2).

221 Haplotype analysis. The markers included in the haplotype analysis were rs368234815 and the 222 ten candidate variants (Figure 4, Panel C). Haplotype construction revealed that in the European 223 ancestry individuals all 11 SNPs are within one unique haplotype block of $11 \mathrm{~kb}$ with LD values 224 consistently high $\left(r^{2}>0.89\right)$, except for rs4803222 and rs368234815 with an $r^{2} \sim 0.50$ with the 
225 other variants (Figure 4). Thus, the top SNP of the candidate variants (rs4803221) tags all

226 associated variants in the block except for rs4803222 and rs368234815.

227 We estimated the frequency of the haplotypes based on the boundaries determined in the 228 haplotype blocks for each population. In the European ancestry samples, the 11 markers formed 22924 haplotypes of which four (denoted H1 to H4) had a frequency higher than $2 \%$ so were 230 included in the haplotype association analysis (Supplementary Table 2). H1 (containing the 231 favorable TT allele of rs368234815) was the haplotype with highest prevalence overall and was 232 more frequent in the clearance group $\left(\mathrm{P}\right.$-value $\left.=4.4 \times 10^{-22}\right) . \mathrm{H} 2, \mathrm{H} 3$ and $\mathrm{H} 4$ contained the 233 unfavorable allele $(\Delta \mathrm{G})$ of rs368234815. H3 and H4 and were significantly associated with 234 persistence $(\mathrm{P}$ value $<0.05$. H2 had a low prevalence $(2 \%)$ and was not associated with $\mathrm{HCV}$ 235 clearance $(\mathrm{P}$ value $=0.53)$.

236 In African ancestry individuals, there was unique haplotype block of $5 \mathrm{~Kb}$ containing 9 out of the 23711 variants with LD $r^{2}$ values ranging from 0.99 to 0.03 (Figure 4). In this population rs4803221 238 is able to capture information only from rs8105790, rs66531907, rs12983038, rs8109889 but not 239 from rs8099917 or rs7248668. Similarly, rs8107030, rs368234815 and rs4803222 only capture 240 information from themselves. Similar to the European ancestry population, rs 368234815 had low 241 LD $r^{2}$ values with the ten variants (Figure 4).

242 In African ancestry individuals, 21 haplotypes were present and five with the highest frequency $243(>6 \%)$ were included in the analysis. Haplotypes $\mathrm{H} 1-\mathrm{H} 4$ are similar to those of the European 244 ancestry populations for the shared markers. Similarly, H1 was more frequent in the clearance 245 group $\left(\mathrm{P}\right.$ value $\left.=1.5 \times 10^{-14}\right)$, however, this haplotype had a considerably lower prevalence in this 
246 sample compared to the European ancestry sample (37.5\% vs. $68.1 \%)$, which can be explained 247 by the differences in the allelic frequency of the $\Delta \mathrm{G}$ allele between those samples (Table 1).

248 In the African ancestry sample, $\mathrm{H} 2$ was the predominant haplotype conferring persistence and 249 has a considerably higher prevalence in this African ancestry group (36\% vs 2\% in the European 250 group). $\mathrm{H} 3$ and $\mathrm{H} 4$ were associated with persistence with comparable effect size but lower 251 significance $(\mathrm{P}=0.02)$. In summary, in both sample groups $\mathrm{H1}$ was significantly associated with 252 clearance. However, the predominant haplotypes conferring persistence were different in each 253 sample group (H3 in European ancestry vs. H2 in African ancestry individuals), Supplementary 254 Table 2. Similar results were observed in the haplotype analysis including common candidate 255 variants and functionally relevant variants, except for the separation of H2 in African Ancestry 256 individuals in $\mathrm{H} 2 \mathrm{a}$ and $\mathrm{H} 2 \mathrm{~b}$. $\mathrm{H} 2 \mathrm{~b}$ conserved similar direction and strength of effect than $\mathrm{H} 2$ 257 (Supplementary Table 3).

258 Next, to determine whether an allele or haplotype could "overcome" the unfavorable allele $(\Delta G)$ 259 of rs 368234815 , we restricted our analysis to haplotypes containing this unfavorable allele in the 260 European ancestry group. We found that the haplotypes with the $\mathrm{C}$ allele at rs4803221 were 261 significantly associated with clearance compared to those containing the G allele (OR: 1.7, 95\% 262 CI: $1.3-2.29, \mathrm{P}$ value $=3.6 \times 10^{-5}$, Table 3). This was not observed in African ancestry 263 individuals (OR for haplotype with the $\mathrm{C}$ allele: 1.25 ; 95\% CI: 0.81-1.9, P value $=0.29$ ). 264 Rs4803221 tags rs8099917 and rs7248668 in the European ancestry group but not in the African 265 ancestry group. Similar to rs4803221, the haplotype containing the T allele of rs8099917 (and G 266 allele of rs7248668) is significantly associated with clearance compared to the one containing the 267 G allele of rs8099917 (and A allele of rs7248668: OR: 1.6, 95\% CI: 1.3-2.16, P value: $1.76 x$ 10268 4) in the European ancestry individuals but not in African ancestry individuals (Table 3). These 
269 data are consistent with a main signal being shared across populations driven by one or more

270 functional variants represented by rs368234815, with potential additional contributions from

271 rs4803221, and/or proxies including rs8099917 and rs7248668 in the European ancestry

272 population.

\section{Identification of potential causal variants}

274 Four SNPs were identified as likely functional (posterior probability $>0.1$, Supplementary Table

275 4). The credible set obtained with PAINTOR, determined by 2 out of 4 variants of the credible 276 set (rs368234815 and rs12982533), overlaps with the previously estimated credible set using a 277 larger dataset (3), narrowing the signal to a $7251 \mathrm{bp}$ region (19:39731904-39739155) located $2782368 \mathrm{bp}$ downstream from IFNL3 and extending until exon 1 of IFNL4 (Supplementary Figure 2).

279 The identified region includes rs368234815 which we confirmed as the main driver of the 280 association signal. The variants identified in the fine-mapping credible set overlapped with 281 regulatory regions in hepatocyte cell lines and liver tissue including $\mathrm{CpG}$ sites that are 282 completely or partially methylated, target sites for transcription factors, DNA methylation sites 283 with $50-100 \%$ methylation in those cells, candidate weak enhancers, polycomb repressors and 284 with transcription associated activity (Supplementary Figure 2). Two other variants (rs4803221 285 and rs10612351) also showed posterior probability values $>0.10$ indicating that they might be 286 considered causal even though rs10612351 is not included in calculated $99 \%$ credible set. We 287 considered that these polymorphisms are plausible candidate variants based both on fine288 mapping and regulatory overlap and these results support the findings of the haplotype analysis.

\section{Analysis of functionally relevant variants}


Rs4803217 and rs1176648444 had a difference of 1 and 0 respectively in the counts of the alternative allele between clearance and persistence in the sequenced panel. Rs4803217 showed no association in the imputed dataset after conditioned on rs368234815 (European ancestry conditioned $\mathrm{P}$ value $=0.15$, African ancestry conditioned $\mathrm{P}$ value $=0.3$, Meta-analysis conditioned $\mathrm{P}$ value $=0.08)$. Rs1176648444 was not associated in African Ancestry $(\mathrm{P}$ value $=0.38)$ but interestingly it showed a significant association in individuals of European Ancestry only in the conditioned analysis (Not conditioned $\mathrm{P}$ value $=0.07$; conditioned $\mathrm{P}$ value $=0.00003$, conditioned meta-analysis $\mathrm{P}$ value $=0.06$ ). In the double conditioned analysis with rs368234815 and rs 1176648444, six out of ten variants associated in the single conditioned analysis showed residual association (Supplementary Table 5). Rs368234815TT- Rs4803217C haplotype was significantly associated with clearance compared with the Rs $368234815 \Delta \mathrm{G}-\mathrm{Rs} 4803217 \mathrm{~A}$ in both populations. In African ancestry population the haplotype Rs368234815 $\Delta \mathrm{G}-\mathrm{Rs} 4803217 \mathrm{C}$ was significantly associated with persistence (Supplementary Table 6). On the other hand, in the European ancestry population, the haplotype rs368234815 $\Delta$ G- rs1176648444A (IFN $\lambda 4-S 70$ ) was associated with clearance with an intermediate effect between rs368234815 GG-rs1176648444G

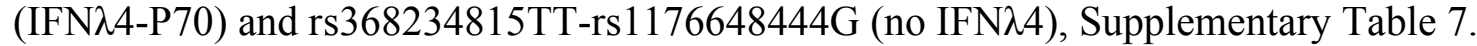

\section{Discussion}

We performed a comprehensive, trans-ethnic analysis of genetic variation in the IFNL region and spontaneous recovery from $\mathrm{HCV}$ infection. We discovered variants with associations independent of the well-described rs368234815 variant that suggest additional genetic contributions to the outcome of this chronic infection.

We observed an rs368234815-independent signal led by rs4803221 (given mainly for the European Ancestry population) and ten other variants in LD including rs8099917 and rs7248668. 
313 In sensitivity analysis we confirmed that this signal was present even after conditioning on 314 rs368234815 and rs1176648444 indicating a residual or modifying effect of the remaining variants. The 315 LD structure of the region in the European ancestry group suggests that the rs 4803221 316 association may be due to any one of a number of variants including rs8099917 and rs7248668.

317 In fact, in the context of haplotypes conferring persistence in this group, the haplotype containing 318 the $\mathrm{C}$ allele of rs4803221, the $\mathrm{T}$ allele of rs8099917 and the $\mathrm{G}$ allele of rs 7248668 were 319 significantly associated with HCV clearance. However, we do not observe a significant signal in 320 individuals of African ancestry at rs4803221, even though its allele frequency and the sample 321 size are similar to the European ancestry group. It is possible that the association of rs 4803221 322 observed in the European ancestry group is explained by linkage with rs8099917 and/or 323 rs7248668, instead of being functional itself. Unfortunately, our power was limited to confirm 324 this inference in the African ancestry group, where we detected an odds ratio of 0.79 with a MAF 325 of 0.06 (power of only 0.38 at a significance level of 0.05 , compared to 1 in the European 326 ancestry group) (27).

327 Rs4803221 is a variant with multiple functions which has been previously linked to $\mathrm{HCV}$ 328 spontaneous clearance in individuals with beta thalassemia (28). The SNP is located in exon one 329 of IFNL4, $357 \mathrm{bp}$ downstream from the transcription start site and $3522 \mathrm{bp}$ upstream from the 330 transcription start site of IFNL3; the $\mathrm{G}$ allele $(\mathrm{MAF}=0.2)$ abolishes a $\mathrm{CpG}$ site and induces a 331 synonymous (Ser $>$ Ser) change at position 30 of the IFNL4 protein. Similar to our findings, Origa 332 et al, found an association with rs4803221 that was independent of rs 12979860 (which is itself in 333 high LD with rs368234815) (28). Rs4803221 significantly improved the viral clearance 334 prediction in patients carrying the un-favorable T allele of rs 12979860 (in high LD with the un335 favorable $\Delta \mathrm{G}$ allele of rs368234815). They hypothesized that the abolishment of methylation 
336 sites might increase expression of IFNL3 and downregulate interferon sensitive genes, reducing 337 net innate antiviral activity (28).

338 A potential 'causal' role has also been described for rs8099917 (1). In a GWAS including 1362 339 European ancestry individuals, G allele was associated with persistence of HCV infection (1). 340 Several specific SNPs were identified as candidates for being causal, however rs368234815 was 341 not described in this panel. In European $\mathrm{HCV}$-infected individuals analyzed for response 342 treatment, haplotypes tagged by the $\mathrm{T}$ allele of rs 8099917 showed higher expression of IFN $\lambda 3$ 343 and IFN $\lambda 2$ but no evaluation was reported on expression of IFN $\lambda 4$ (29). Analogous results were 344 found in a Japanese cohort where the expression IFNL3 and IFNL2 mRNA was lower in the 345 carriers of the $\mathrm{G}$ allele (30). In our current study, $\mathrm{T}$ allele is associated with clearance in the 346 context of the $\Delta \mathrm{G}$ allele of rs368234815, which corresponds with IFNL4 transcription but HCV 347 persistence (4). Rs8099917 is located $8.9 \mathrm{~kb}$ upstream from IFNL3 and $16 \mathrm{~kb}$ upstream from 348 IFNL2. If we assume the model that rs368234815 regulates the expression of IFN $\lambda 4$ (Figure 1), 349 it would be worthwhile to investigate if the statistically independent effect of rs 8099917 350 observed in this study is perhaps caused by an increase in expression of IFN $\lambda 3$ and IFN $\lambda 2$, a 351 decrease in the production of IFN $\lambda 4$ in those individuals with the $\Delta \mathrm{G}$ allele at rs368234815, or 352 both.

353 SNP rs7248668 located in the 5' region of IFNL4 is in high LD with rs8099917 in populations 354 included in this analysis and in The 1000 Genomes Project independently of ancestry (14). In 355 fine mapping analysis, the haplotype containing the $\mathrm{G}$ allele was associated with virologic 356 response to pegylated interferon- $\alpha$ and ribavirin therapy for chronic hepatitis $\mathrm{C}$ in a Japanese 357 population (30). Similarly, the patients with the GG genotype showed virologic response rates up 358 to four times higher than those for patients with unfavorable genotypes in $\mathrm{HIV} / \mathrm{HCV}$ co-infected 
359 patients of European ancestry (31). In our study the same $\mathrm{G}$ allele is associated with spontaneous

360 clearance; even though the phenotypes are not completely comparable, in general the G allele

361 favors the clearance of the virus in each context across studies. Due to their high LD the effect of

362 rs7248668 is not separable from that of rs8099917.

363 Our fine mapping analysis using PAINTOR indicates that the potential causal variant in this

364 locus is contained in the IFNL3-IFNL4 gene region. This credible set informed by our analysis

365 harbors the compound di-nucleotide exonic variant (rs368234815, $\Delta \mathrm{G} / \mathrm{TT}$ ) and the rs 4803221

366 variant, but does not contain rs 8099917 or rs 7248668 . It is possible that we did not find a high

367 posterior probability for those 2 latter SNPs because they are in high LD with rs4803221 in

368 European ancestry subjects, where the significant independent effect was observed. We consider

369 that the expansion of the sample size of African ancestry individuals could allow

370 disentanglement of the effects of rs4803221, rs8099917 and rs7248668. The coding nature of the

371 rs368234815, the high significance and large effect-size, and the low LD between this variant

372 and the others in the region (especially in the African ancestry population) contributed to

373 determine this variant as functionally relevant.

374 The identification of rs12982533 as functionally relevant deserves further analysis. This variant

375 has been included as part of haplotypes associated with response to treatment $(13,32)$ but not

376 with spontaneous HCV clearance; it is located 3.7kb 3' of IFNL3 and its functional role is

377 unknown. It is important to notice that we limited this analysis to only variants that were

378 consistently present in both ancestry groups and it is possible that this set of variants fails to 379 capture putatively important variation within or around the IFNL locus. 
The results of the of rs368234815-rs1176648444 haplotype analysis in European ancestry agree with findings previously described by the Swiss Hepatitis Cohort Study Group (33) where they demonstrated that individuals with IFN $\lambda 4-\mathrm{S} 70$ have rates of $\mathrm{HCV}$ clearance that are intermediate to those with IFN $\lambda 4$-P70 and those with rs368234815TT/TT genotype, who do not produce the IFN $\lambda 4$ protein. Similarly, our findings on rs368234815-rs4803217 haplotype are in concordance with the association of rs368234815 $\Delta \mathrm{G}$ : rs4803217G with the poorest virologic response to peg- interferon alpha and ribavirin therapy in African Americans (34); even though it is not the same phenotype, it suggests an interaction of the two variants responsible for lower rate of resolution of the infection in general. The restriction of the haplotype effect to specific populations deserves further analysis including a larger sample capable of capturing all haplotype diversity.

One strength of this analysis is the sequencing strategy which allowed us to unambiguously map read pairs to specific segments of the IFNL region, and call the genetic variants with higher accuracy than using conventional methods of short-read sequencing. The "conditioned by design" composition of the panel with concordant and discordant individuals enabled the detection of variants conferring an effect on HCV clearance that is adjusted for the allele present at rs368234815. Even though the sample size of the sequencing panel is small, its particular configuration makes it suitable to detect variants with a large effect. The findings from this panel were supported by the results of the statistically conditioned analysis in a much larger sample size with similar characteristics adding reliability to the findings. One limitation of the study is that we established a rather high cut off for the selection of the variants with differences in the allele count since the size of the panel precluded the evaluation of rare variants using standard 
402 statistical tests and we excluded rare variants in the imputation panel since the imputation quality

403 is usually low for those variants and any derived results would be considered uncertain.

404 In this study we fine-mapped the IFNL region and found results that support an independent

405 genetic effect of several variants in this locus. Our results are applicable to the European ancestry

406 population with our current sample sizes and are hypothesis-generating regarding additional

407 factors contributing to the higher clearance in European ancestry and African ancestry

408 individuals. Our findings are relevant and complementary to previous analyses aimed to

409 understand the genetic basis of HCV clearance and the differences in the immune response to

410 this infection across populations.

\section{References}

412 (1) Rauch A, Kutalik Z, Descombes P, Cai T, Di Iulio J, Mueller T, et al. Genetic variation in

413 IL28B is associated with chronic hepatitis C and treatment failure: a genome-wide association

414 study. Gastroenterology 2010 Apr;138(4):1338-45, 1345.e1-7.

415 (2) Duggal P, Thio CL, Wojcik GL, Goedert JJ, Mangia A, Latanich R, et al. Genome-wide 416 association study of spontaneous resolution of hepatitis $\mathrm{C}$ virus infection: data from multiple 417 cohorts. Ann Intern Med 2013 Feb 19;158(4):235-245.

418 (3) Vergara C, Thio CL, Johnson E, Kral AH, O'Brien TR, Goedert JJ, et al. Multi-Ancestry

419 Genome-Wide Association Study of Spontaneous Clearance of Hepatitis C Virus.

420 Gastroenterology 2018 Dec 26. 
421 (4) Prokunina-Olsson L, Muchmore B, Tang W, Pfeiffer RM, Park H, Dickensheets H, et al. A 422 variant upstream of IFNL3 (IL28B) creating a new interferon gene IFNL4 is associated with 423 impaired clearance of hepatitis C virus. Nat Genet 2013 Feb;45(2):164-171.

424 (5) Hong M, Schwerk J, Lim C, Kell A, Jarret A, Pangallo J, et al. Interferon lambda 4 425 expression is suppressed by the host during viral infection. J Exp Med 2016 Nov $426 \quad 14 ; 213(12): 2539-2552$.

427 (6) Thomas DL, Thio CL, Martin MP, Qi Y, Ge D, O'Huigin C, et al. Genetic variation in IL28B 428 and spontaneous clearance of hepatitis C virus. Nature 2009 Oct 8;461(7265):798-801.

429 (7) Tillmann HL, Thompson AJ, Patel K, Wiese M, Tenckhoff H, Nischalke HD, et al. A 430 polymorphism near IL28B is associated with spontaneous clearance of acute hepatitis C virus 431 and jaundice. Gastroenterology 2010 Nov;139(5):1586-92, 1592.e1.

432 (8) Knapp S, Warshow U, Ho KM, Hegazy D, Little AM, Fowell A, et al. A polymorphism in 433 IL28B distinguishes exposed, uninfected individuals from spontaneous resolvers of HCV 434 infection. Gastroenterology $2011 \mathrm{Jul} ; 141(1): 320-5,325 . e 1-2$.

435 (9) Aka PV, Kuniholm MH, Pfeiffer RM, Wang AS, Tang W, Chen S, et al. Association of the 436 IFNL4-DeltaG Allele With Impaired Spontaneous Clearance of Hepatitis C Virus. J Infect Dis $437 \quad 2014$ Feb 1;209(3):350-354.

438 (10) Chinnaswamy S. Genetic variants at the IFNL3 locus and their association with hepatitis C 439 virus infections reveal novel insights into host-virus interactions. J Interferon Cytokine Res 2014 $440 \quad$ Jul;34(7):479-497. 
441 (11) Spain SL, Barrett JC. Strategies for fine-mapping complex traits. Hum Mol Genet 2015 Oct $442 \quad 15 ; 24(\mathrm{R} 1): \mathrm{R} 111-9$.

443 (12) Mandelker D, Schmidt RJ, Ankala A, McDonald Gibson K, Bowser M, Sharma H, et al. 444 Navigating highly homologous genes in a molecular diagnostic setting: a resource for clinical 445 next-generation sequencing. Genet Med 2016 Dec;18(12):1282-1289.

446 (13) Smith KR, Suppiah V, O'Connor K, Berg T, Weltman M, Abate ML, et al. Identification of 447 improved IL28B SNPs and haplotypes for prediction of drug response in treatment of hepatitis C 448 using massively parallel sequencing in a cross-sectional European cohort. Genome Med 2011 $449 \quad$ Aug 31;3(8):57.

450 (14) Sudmant PH, Rausch T, Gardner EJ, Handsaker RE, Abyzov A, Huddleston J, et al. An 451 integrated map of structural variation in 2,504 human genomes. Nature 2015 Oct $452 \quad 1 ; 526(7571): 75-81$.

453 (15) Kotenko SV, Gallagher G, Baurin VV, Lewis-Antes A, Shen M, Shah NK, et al. IFN454 lambdas mediate antiviral protection through a distinct class II cytokine receptor complex. Nat 455 Immunol 2003 Jan;4(1):69-77.

456 (16) Sheppard P, Kindsvogel W, Xu W, Henderson K, Schlutsmeyer S, Whitmore TE, et al. IL457 28, IL-29 and their class II cytokine receptor IL-28R. Nat Immunol 2003 Jan;4(1):63-68.

458 (17) O'Brien TR, Prokunina-Olsson L, Donnelly RP. IFN-lambda4: the paradoxical new member 459 of the interferon lambda family. J Interferon Cytokine Res 2014 Nov;34(11):829-838. 
460 (18) Wojcik GL, Thio CL, Kao WH, Latanich R, Goedert JJ, Mehta SH, et al. Admixture 461 analysis of spontaneous hepatitis C virus clearance in individuals of African descent. Genes 462 Immun 2014 Apr;15(4):241-246.

463 (19) Li Y, Willer C, Sanna S, Abecasis G. Genotype imputation. Annu Rev Genomics Hum 464 Genet 2009;10:387-406.

465 (20) Willer CJ, Li Y, Abecasis GR. METAL: fast and efficient meta-analysis of genomewide 466 association scans. Bioinformatics 2010 Sep 1;26(17):2190-2191.

467 (21) Schaid DJ, Chen W, Larson NB. From genome-wide associations to candidate causal 468 variants by statistical fine-mapping. Nat Rev Genet 2018 Aug;19(8):491-504.

469 (22) Gabriel SB, Schaffner SF, Nguyen H, Moore JM, Roy J, Blumenstiel B, et al. The structure 470 of haplotype blocks in the human genome. Science 2002 Jun 21;296(5576):2225-2229.

471 (23) Barrett JC, Fry B, Maller J, Daly MJ. Haploview: analysis and visualization of LD and 472 haplotype maps. Bioinformatics 2005 Jan 15;21(2):263-265.

473 (24) Schaid DJ, Rowland CM, Tines DE, Jacobson RM, Poland GA. Score tests for association 474 between traits and haplotypes when linkage phase is ambiguous. Am J Hum Genet 2002 475 Feb;70(2):425-434.

476 (25) Kichaev G, Pasaniuc B. Leveraging Functional-Annotation Data in Trans-ethnic Fine477 Mapping Studies. Am J Hum Genet 2015 Aug 6;97(2):260-271. 
478 (26) Kichaev G, Yang WY, Lindstrom S, Hormozdiari F, Eskin E, Price AL, et al. Integrating

479 functional data to prioritize causal variants in statistical fine-mapping studies. PLoS Genet 2014

480 Oct 30;10(10):e1004722.

481 (27) Purcell S, Cherny SS, Sham PC. Genetic Power Calculator: design of linkage and

482 association genetic mapping studies of complex traits. Bioinformatics 2003 Jan;19(1):149-150.

483 (28) Origa R, Marceddu G, Danjou F, Perseu L, Satta S, Demartis FR, et al. IFNL3

484 polymorphisms and HCV infection in patients with beta thalassemia. Ann Hepatol 2015 May-

485 Jun;14(3):389-395.

486 (29) Suppiah V, Moldovan M, Ahlenstiel G, Berg T, Weltman M, Abate ML, et al. IL28B is

487 associated with response to chronic hepatitis $\mathrm{C}$ interferon-alpha and ribavirin therapy. Nat Genet

4882009 Oct;41(10):1100-1104.

489 (30) Tanaka Y, Nishida N, Sugiyama M, Kurosaki M, Matsuura K, Sakamoto N, et al. Genome490 wide association of IL28B with response to pegylated interferon-alpha and ribavirin therapy for 491 chronic hepatitis C. Nat Genet 2009 Oct;41(10):1105-1109.

492 (31) Fernandez-Rodriguez A, Rallon N, Berenguer J, Jimenez-Sousa MA, Cosin J, Guzman-

493 Fulgencio M, et al. Analysis of IL28B alleles with virologic response patterns and plasma

494 cytokine levels in HIV/HCV-coinfected patients. AIDS 2013 Jan 14;27(2):163-173.

495 (32) Booth DR, Ahlenstiel G, George J. Pharmacogenomics of hepatitis C infections:

496 personalizing therapy. Genome Med 2012 Dec 26;4(12):99. 
497 (33) Terczynska-Dyla E, Bibert S, Duong FH, Krol I, Jorgensen S, Collinet E, et al. Reduced 498 IFNlambda4 activity is associated with improved HCV clearance and reduced expression of 499 interferon-stimulated genes. Nat Commun 2014 Dec 23;5:5699.

500 (34) O'Brien TR, Pfeiffer RM, Paquin A, Lang Kuhs KA, Chen S, Bonkovsky HL, et al.

501 Comparison of functional variants in IFNL4 and $\lambda \mathrm{L} 3$ for association with HCV clearance. J 502 Hepatol 2015 Nov;63(5):1103-1110.

504 Acknowledgments: We thank the participants of the study. We also thank Cristian Velarde for 505 graphic design of the Figures.

\section{$506 \quad$ Legends of Tables}

507 Table 1. Genetic ancestry, HCV status and rs368234815 genotype distribution of the analyzed 508 individuals.

509 Table 2. Variants with a difference $\geq 5$ in alternative allele count in sequenced individuals and 510 replication in the meta-analysis of the association test of imputed variants in the IFNL region 511 conditioned on the rs368234815 genotype. Bold text indicates positions with meta-analysis $512 \mathrm{p}<0.05$ from imputed data.

513 Table 3. Association analysis of the 2 variant haplotypes (rs4803221-rs368234815 and 514 rs368234815- rs8099917) in individuals carrying the $\Delta \mathrm{G}$ allele of rs368234815.

\section{$515 \quad$ Legends of Figures}


516 Figure 1. Top panel: Depiction of putative role of rs368234815 genotypes in HCV persistence or

517 clearance. Bottom panel: Schematic representation of concordant and discordant panels of

518 individuals used in the sequencing analysis.

519 Figure 2. Schematic representation of the fine mapping analysis performed in this study.

520 Figure 3. Genetic structure of the IFNL locus, amplified fragments used for targeted sequencing,

521 and two main variants associated in prior GWAS studies with HCV clearance (rs12979860 and

522 rs368234815). Genetic coordinates are based on The Genome Reference Consortium Human

523 build 37 (GRCh37/hg19).

524 Figure 4. Results of the analysis conditioned on rs368234815 and LD patterns of the top 525 associated variants from that analysis. A) Meta-analysis conditioned on rs368234815 genotype. 526 Variants represented in squares in panel B are the 23 of 25 variants that showed a difference of at 527 least 5 in counts between clearance and persistence groups in the sequencing analysis. 528 Recombination in this region is plotted in the background in light blue. Pairwise LD between the 529 top associated variant and other variants in the region were estimated using LD data in the 530 European (EUR) population in the 1000 Genomes project (hg19/Nov 2014). The color from blue 531 to red represents the $r^{2}$ values relative to the peak position after conditioning, rs4803221. B) P 532 values of rs368234815 and the 10 SNPs with remaining significance in the conditional analysis 533 and their location on the genes in the region. C) LD plot of those variants in individuals of 534 European and African ancestry in the genotyped/imputed dataset. The value within each 535 diamond of the LD plot represents the pairwise correlation between tagging SNPs defined by 536 sides of each the diamond. Shading represents the magnitude and significance of pairwise LD 537 represented by the $r^{2}$ value, with a red- yellow gradient reflecting higher to lower LD values. 
538 Association plots were graphed with Locus Zoom, P value and LD plots were generated using 539 the package snp.plotter implemented in R. 


\begin{tabular}{|c|c|c|c|c|c|c|c|c|}
\hline \multirow{3}{*}{ Ancestry group } & \multirow{3}{*}{ HCV status } & \multicolumn{3}{|c|}{ Sequencing analysis $(N=64)$} & \multicolumn{4}{|c|}{ Imputation analysis $(\mathrm{N}=3552)$} \\
\hline & & \multicolumn{2}{|c|}{$\begin{array}{c}\text { Genotype at } \\
\text { rs368234815 (n) }\end{array}$} & \multirow{2}{*}{ Total } & \multicolumn{3}{|c|}{ Genotype at rs368234815 (n) } & \multirow{2}{*}{ Tota } \\
\hline & & $\Delta G / \Delta G$ & TT/TT & & $\Delta G / \Delta G$ & $\mathbf{T T} / \Delta \mathrm{G}$ & TT/TT & \\
\hline \multirow{3}{*}{ African Ancestry } & Clearance & 13 & 8 & 21 & 91 & 137 & 92 & 320 \\
\hline & Persistence & 13 & 11 & 24 & 626 & 742 & 147 & 1515 \\
\hline & Total & 26 & 19 & 45 & 717 & 879 & 239 & 1835 \\
\hline \multirow{3}{*}{ European Ancestry } & Clearance & 1 & 8 & 9 & 39 & 232 & 421 & 692 \\
\hline & Persistence & 2 & 8 & 10 & 128 & 521 & 376 & 1025 \\
\hline & Total & 3 & 16 & 19 & 167 & 753 & 797 & 1717 \\
\hline
\end{tabular}




\begin{tabular}{|c|c|c|c|c|c|c|c|c|c|c|c|c|c|}
\hline \multirow[b]{3}{*}{ rsID } & \multirow{2}{*}{\multicolumn{3}{|c|}{ SNP }} & \multirow{2}{*}{\multicolumn{3}{|c|}{$\begin{array}{c}\text { Analysis of individuals in } \\
\text { sequencing panel }(\mathrm{N}=64) \\
\text { Counts of Alternative } \\
\text { allele }\end{array}$}} & \multicolumn{7}{|c|}{ Analysis of imputed data conditioned on rs368234815 } \\
\hline & & & & & & & \multicolumn{3}{|c|}{$\begin{array}{l}\text { European Ancestry population } \\
\qquad(\mathrm{N}=1,717)\end{array}$} & \multicolumn{3}{|c|}{$\begin{array}{c}\text { African Ancestry } \\
\text { Population } \\
(\mathbf{N}=1,835) \\
\end{array}$} & \multirow{2}{*}{$\begin{array}{c}\begin{array}{c}\text { Meta- } \\
\text { analysis } \\
(\mathrm{N}=3,552)\end{array} \\
\text { P value }\end{array}$} \\
\hline & Position & Ref & Alt & Clear & Persist & Diff. & Freq. & OR & P Value & Freq. & OR & P Value & \\
\hline rs8107090 & 39721915 & $\mathrm{~T}$ & A & 19 & 24 & -5 & 0.40 & 0.96 & 0.67 & 0.57 & 0.90 & 0.45 & 0.400 \\
\hline rs35408086 & 39726810 & G & A & 11 & 19 & -8 & 0.39 & 0.98 & 0.80 & 0.21 & 0.95 & 0.66 & 0.621 \\
\hline rs11883239 & 39727480 & G & A & 6 & 17 & -11 & 0.39 & 0.98 & 0.80 & 0.13 & 0.83 & 0.21 & 0.287 \\
\hline rs11883201 & 39727490 & $\mathrm{~A}$ & $\mathrm{G}$ & 19 & 24 & -5 & 0.40 & 0.97 & 0.69 & 0.59 & 0.93 & 0.59 & 0.507 \\
\hline rs955155 & 39729479 & $\mathrm{G}$ & A & 2 & 8 & -6 & 0.26 & 0.98 & 0.84 & 0.07 & 0.86 & 0.41 & 0.459 \\
\hline rs12609937 & 39731204 & $\mathrm{~A}$ & $\mathrm{G}$ & 28 & 35 & -7 & 0.91 & 0.95 & 0.72 & 0.98 & 0.99 & 0.97 & 0.786 \\
\hline rs115166799 & 39732212 & A & G & 12 & 6 & 6 & N/A & N/A & N/A & 0.19 & 0.98 & 0.88 & 0.882 \\
\hline rs8105790 & 39732501 & $\mathbf{T}$ & C & 6 & 13 & -7 & 0.20 & 0.53 & $3.21 \times 10^{-05}$ & 0.19 & 0.94 & 0.64 & 0.001 \\
\hline rs8102358 & 39735012 & G & A & 13 & 7 & 6 & NA & N/A & NA & 0.25 & 1.03 & 0.82 & 0.820 \\
\hline rs8107030 & 39736719 & A & G & 0 & 7 & -7 & 0.19 & 0.54 & $4.84 \times 10^{-05}$ & 0.04 & 0.91 & 0.72 & 0.002 \\
\hline rs12971396 & 39737866 & $\mathrm{C}$ & G & 7 & 13 & -6 & 0.20 & 0.53 & $2.79 \times 10^{-05}$ & 0.19 & 0.94 & 0.68 & 0.001 \\
\hline rs4803221 & 39739129 & C & G & 7 & 13 & -6 & 0.20 & 0.51 & $7.49 \times 10^{-06}$ & 0.19 & 0.93 & 0.60 & $4.86 \times 10^{-04}$ \\
\hline rs73555604 & 39739170 & $\mathrm{C}$ & $\mathrm{T}$ & 12 & 6 & 6 & 0.01 & 1.31 & 0.47 & 0.22 & 1.01 & 0.97 & 0.597 \\
\hline rs4803222 & 39739353 & G & $\mathrm{C}$ & 9 & 14 & -5 & 0.30 & 0.55 & 0.06 & 0.27 & 0.85 & 0.23 & 0.029 \\
\hline rs66531907 & 39740675 & $\mathrm{C}$ & A & 6 & 12 & -6 & 0.19 & 0.55 & $5.90 \times 10^{-05}$ & 0.19 & 0.90 & 0.48 & $9.57 \times 10^{-04}$ \\
\hline rs12983038 & 39741124 & G & A & 6 & 11 & -5 & 0.19 & 0.54 & $3.22 \times 10^{-05}$ & 0.19 & 0.91 & 0.54 & $8.75 \times 10^{-04}$ \\
\hline rs8109889 & 39742770 & $\mathrm{C}$ & $\mathbf{T}$ & 6 & 12 & -6 & 0.19 & 0.57 & $1.01 \times 10^{-04}$ & 0.19 & 0.90 & 0.46 & 0.001 \\
\hline rs8099917 & 39743165 & $\mathbf{T}$ & G & $\mathbf{0}$ & 5 & -5 & 0.19 & 0.57 & $1.22 \times 10^{-04}$ & 0.06 & 0.79 & 0.28 & $5.54 \times 10^{-04}$ \\
\hline rs7248668 & 39743821 & G & A & $\mathbf{0}$ & 5 & -5 & 0.19 & 0.57 & $9.41 \times 10^{-05}$ & 0.06 & 0.79 & 0.29 & $5.14 \times 10^{-04}$ \\
\hline rs10853728 & 39745146 & $\mathrm{C}$ & $G$ & 26 & 34 & -8 & 0.65 & 0.94 & 0.58 & 0.74 & 0.85 & 0.18 & 0.177 \\
\hline rs10775535 & 39745181 & $\mathrm{C}$ & $\mathrm{T}$ & 29 & 34 & -5 & N/A & N/A & N/A & N/A & N/A & N/A & N/A \\
\hline rs56116812 & 39747090 & G & A & 11 & 18 & -7 & 0.12 & 1.34 & 0.06 & 0.23 & 0.90 & 0.41 & 0.473 \\
\hline rs116236518 & 39749790 & $\mathrm{C}$ & $\mathrm{T}$ & 5 & 0 & 5 & N/A & N/A & N/A & 0.02 & 1.48 & 0.26 & 0.262 \\
\hline rs10424607 & 39749922 & $\mathrm{~A}$ & $\mathrm{C}$ & 18 & 23 & -5 & 0.29 & 1.04 & 0.81 & 0.51 & 0.98 & 0.86 & 0.966 \\
\hline rs251908 & 39764449 & $\mathrm{~A}$ & $\mathrm{G}$ & 30 & 35 & -5 & N/A & N/A & N/A & N/A & N/A & N/A & N/A \\
\hline
\end{tabular}




\begin{tabular}{|c|c|c|c|c|c|}
\hline \multicolumn{6}{|c|}{$\begin{array}{c}\text { European Ancestry } \\
\text { (Number of haplotypes= 1087) }\end{array}$} \\
\hline \multicolumn{3}{|c|}{ Haplotype rs4803221- rs368234815 } & $\begin{array}{c}\text { Frequency in Clearance } \\
\text { (Number of haplotypes }=310 \text { ) }\end{array}$ & $\begin{array}{c}\text { Frequency in Persistence } \\
\text { (Number of haplotypes }=777 \text { ) }\end{array}$ & OR (95\% CI, P value) \\
\hline G- $\Delta \mathrm{G}$ Haplotype & G & $\Delta \mathrm{G}$ & 0.53 & 0.66 & 1 \\
\hline C- $\Delta \mathrm{G}$ Haplotype & $\mathrm{C}$ & $\Delta \mathrm{G}$ & 0.47 & 0.34 & $1.7(1.3-2.29,3.6 x 10-5)$ \\
\hline \multicolumn{6}{|c|}{ Haplotype rs368234815- rs8099917 } \\
\hline$\Delta \mathrm{G}-\mathrm{G}$ Haplotype & $\Delta \mathrm{G}$ & G & 0.51 & 0.63 & 1 \\
\hline$\Delta \mathrm{G}-\mathrm{T}$ Haplotype & $\Delta \mathrm{G}$ & $\mathrm{T}$ & 0.49 & 0.37 & $1.6(1.3-2.16,1.76 \times 10-4)$ \\
\hline \multicolumn{6}{|c|}{$\begin{array}{c}\text { African Ancestry } \\
\text { (Number of haplotypes= 2333) }\end{array}$} \\
\hline \multicolumn{3}{|c|}{ Haplotype rs4803221- rs368234815 } & $\begin{array}{c}\text { Frequency in Clearance } \\
\text { (Number of haplotypes= } 319 \text { ) }\end{array}$ & $\begin{array}{c}\text { Frequency in Persistence } \\
\text { (Number of haplotypes= } 1994\end{array}$ & OR (95\% CI, P value) \\
\hline G- $\Delta \mathrm{G}$ Haplotype & G & $\Delta \mathrm{G}$ & 0.29 & 0.30 & 1 \\
\hline C- $\Delta \mathrm{G}$ Haplotype & $\mathrm{C}$ & $\Delta \mathrm{G}$ & 0.71 & 0.71 & $1.02(0.78-1.32,0.88)$ \\
\hline \multicolumn{6}{|c|}{ Haplotype rs368234815- rs8099917 } \\
\hline$\Delta \mathrm{G}-\mathrm{G}$ Haplotype & $\Delta \mathrm{G}$ & G & 0.08 & 0.10 & 1 \\
\hline$\Delta \mathrm{G}-\mathrm{T}$ Haplotype & $\Delta \mathrm{G}$ & $\mathrm{T}$ & 0.92 & 0.90 & $1.25(0.81-1.9,0.29)$ \\
\hline
\end{tabular}




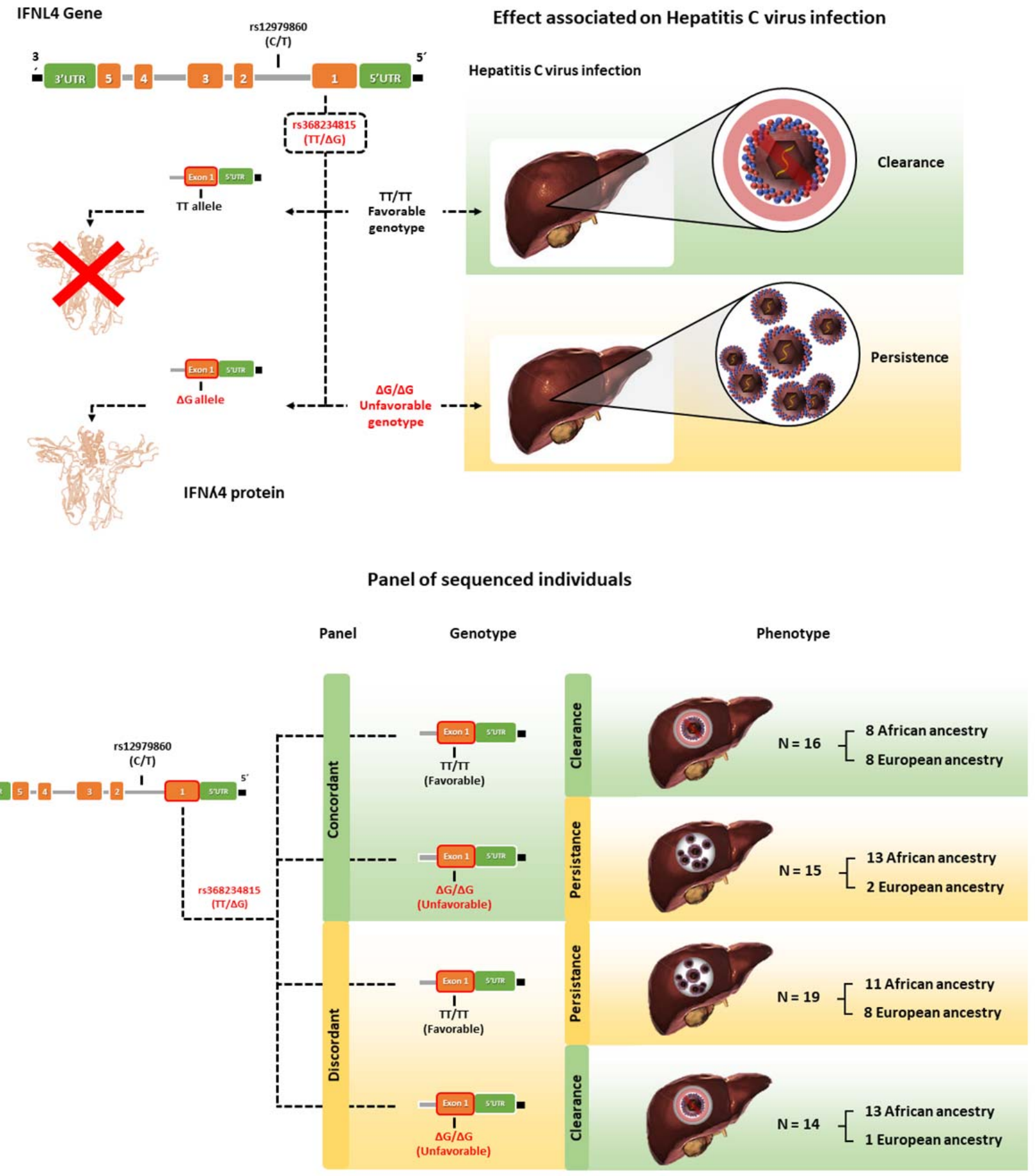




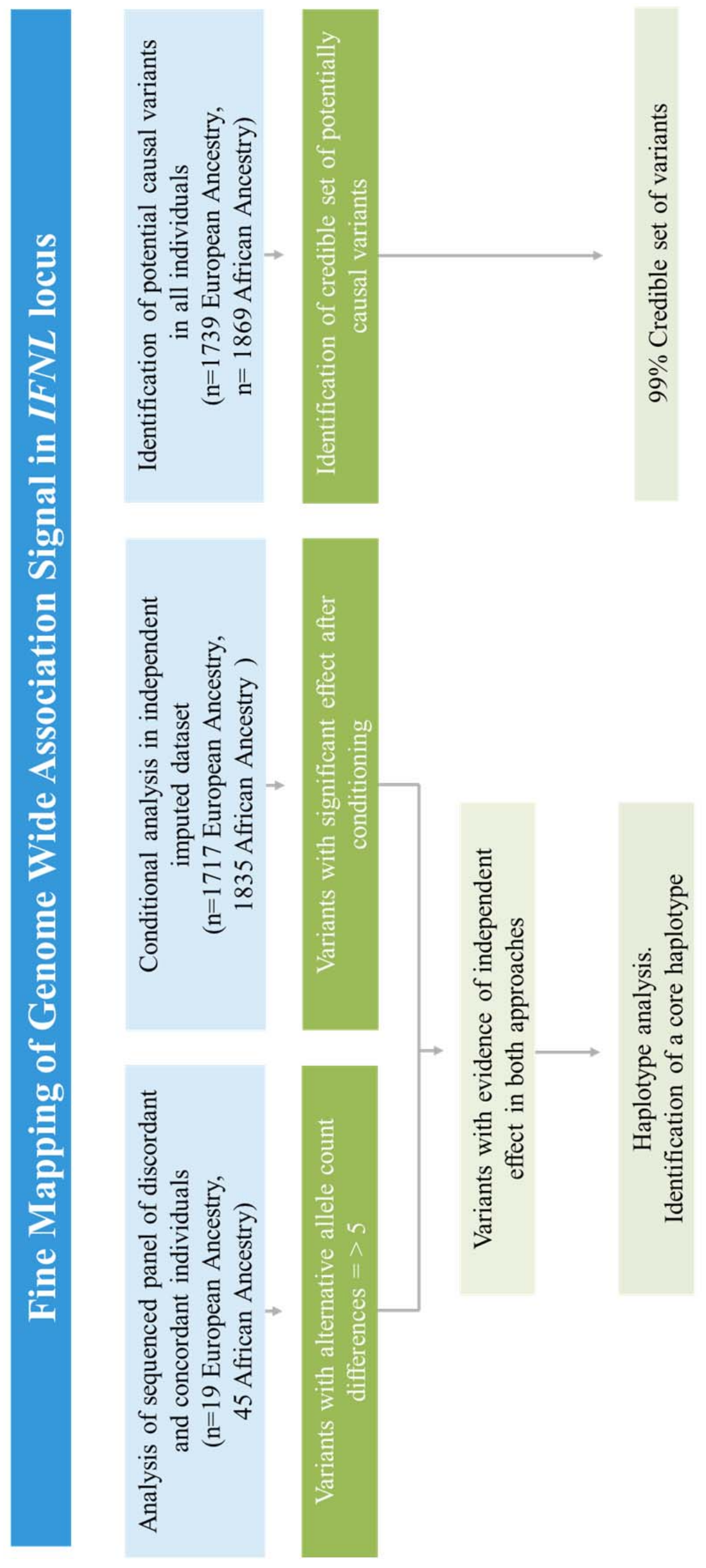




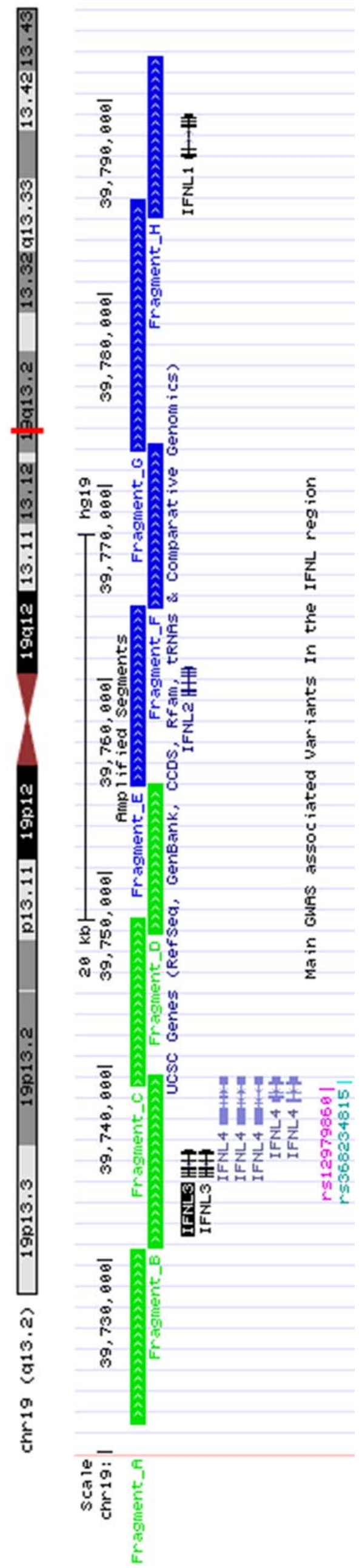



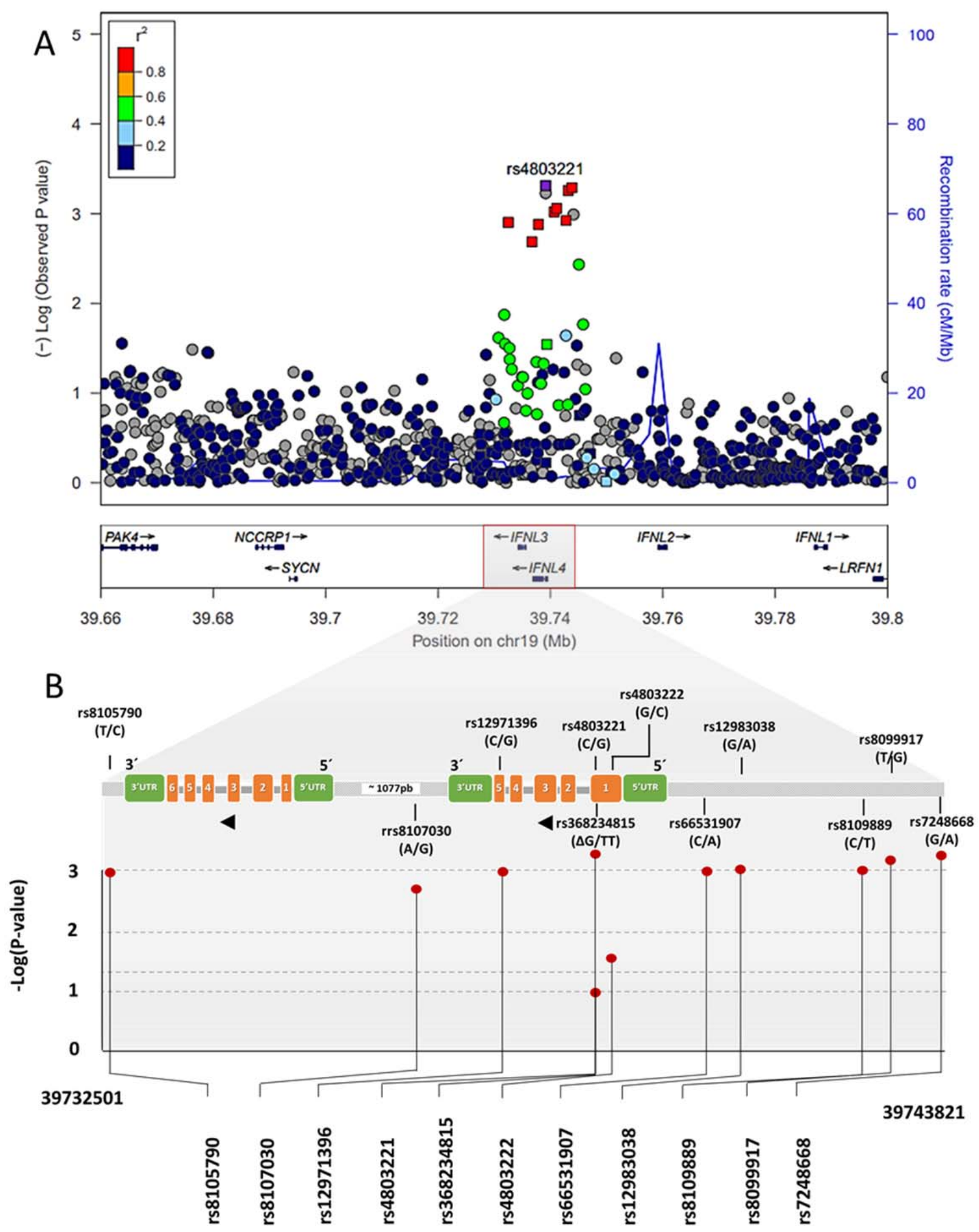
C

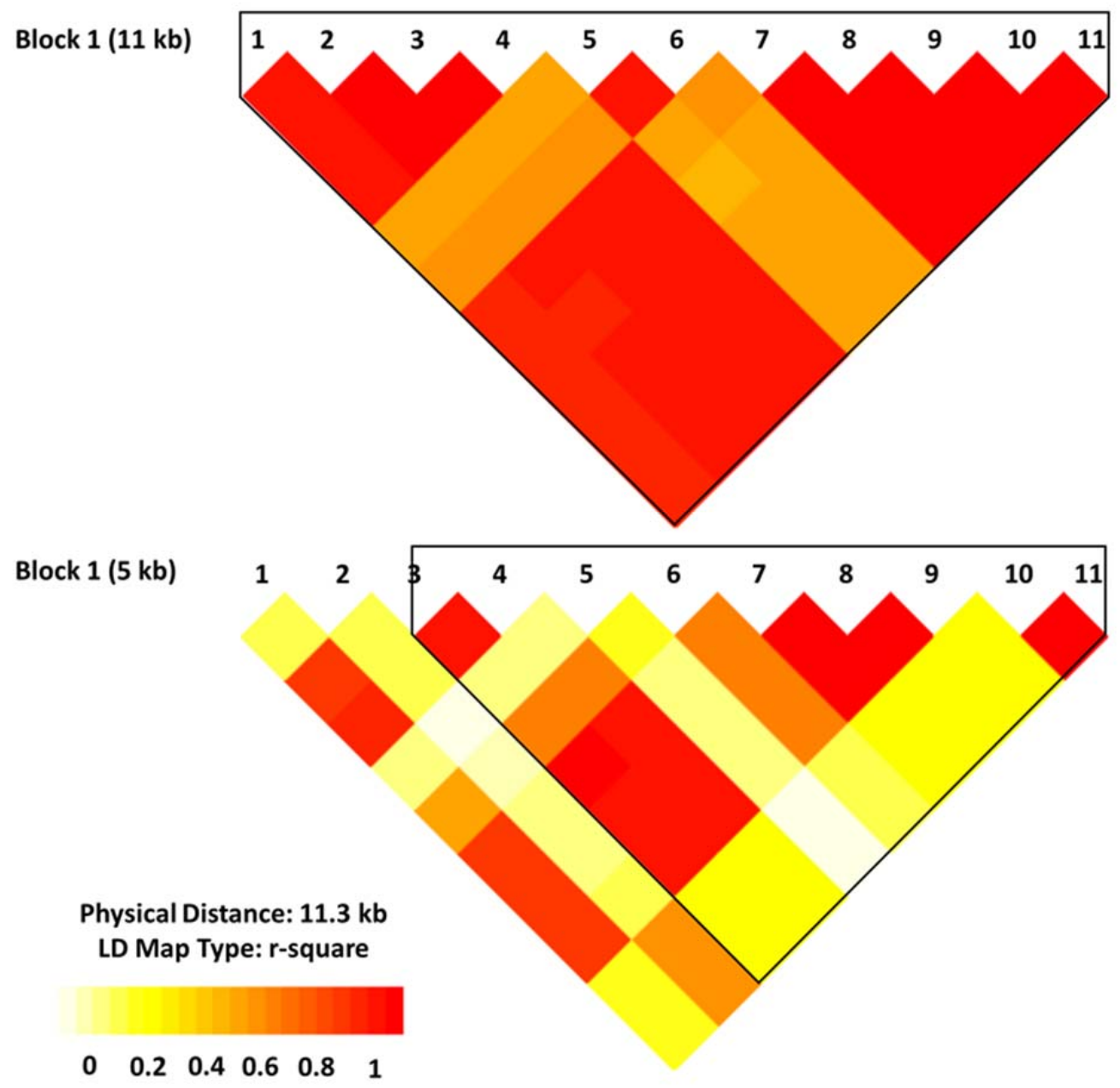

